\title{
On the use of a linear interpolation method in the measurement procedure of a seven-hole pressure probe
}

\author{
M.C. Gameiro Silva ${ }^{a, *}$, C.A.C. Pereira ${ }^{b}$, J.M.S. Cruz ${ }^{c}$ \\ a Departamento de Engenharia Mecânica, Faculdade de Ciências e Tecnologia, Universidade de Coimbra, \\ Pólo II, 3030 Coimbra, Portugal \\ ${ }^{\mathrm{b}}$ Departmento de Engenharia Mecânica, Escola Sup. de Tecnologia e Gestão, Instituto Politécnico de Viseu, Viseu, Portugal \\ ${ }^{\mathrm{c}}$ Escola Secundária Bernardino Machado, 3080 Figueira da Foz, Portugal
}

Received 8 January 2002; accepted 10 March 2003

\begin{abstract}
Calibration and measurement procedures implemented to work with a seven-hole directional pressure probe in the mapping of pressure and velocity wind tunnel flows are reported in this paper. The performances of two different methods used to derive the flow characteristics (direction and magnitude of flow velocity vector and total and dynamic pressure) from the seven pressure values measured by the probe, are comparatively evaluated. The conventional method, based upon the fitting of the calibration directional data with fourth order polynomial functions is compared with a new method where direct linear interpolation is performed over the calibration matrices. Besides the comparison of the two methods, the fundamentals of the working procedure of the probe are briefly explained and an example of the mapping of the flow field around a wing model in a wind tunnel experiment is presented.
\end{abstract}

(C) 2003 Elsevier Inc. All rights reserved.

Keywords: Seven-hole pressure probe; Flow field mapping; Wind tunnel measurements

\section{Introduction}

The seven hole probe is a pressure measurement sensor, which was firstly presented in 1980 by Gallington and Sisson [1]. It has the capability of providing quantitative information about the three components of the flow velocity vector, as well as about the total and dynamic pressures. Its simple shape, relatively easy construction and low cost turned it in one of the most used techniques for velocity and pressure fields surveys on wind tunnel tests. In the literature, a large number of papers where the experimental results were obtained using this measurement technique can be found, e.g. those reported by Hackett and Sugavanam [2], Hackett et al. [3], Coggotti [4,5] and Silva and Viegas [6].

In the first directional pressure probes, the determination of the flow direction was based upon the equalization of two or more pressure taps placed in symmetrical positions, which resulted in a very time

\footnotetext{
${ }^{*}$ Corresponding author. Fax: +351-239-790-771.

E-mail address: manuel.gameiro@dem.uc.pt (M.C. Gameiro Silva).
}

consuming measurement process. Besides, the flow sensitivity solid angle was reduced $\left( \pm 30^{\circ}\right)$, thus implying that, in many cases, the flow velocity vector could not be determined because it was out of the probe measuring spatial zone. Those problems were overcome, firstly with the introduction of non-nulling calibration methods (Ostowari and Wentz [7]), and secondly with the sevenhole geometry that allowed, with the quite ingenious measuring method proposed by Gallington and Sisson [1], the increase of the sensitivity solid angle until values around $70^{\circ}$. To reduce the blind part of the probe, a new geometry using two seven-hole probes in opposite senses was proposed, resulting on the fourteen-hole probe (Cogotti [5]).

The seven-hole probe is composed of a cylindrical tube with a conical or hemispherical extremity, where seven holes are drilled, one in the centre and the remaining six placed around it on an axi-symmetrical arrangement. Each hole is a pressure tap connected to a measuring device. More details about the geometry and construction process can be found in Everett et al. [8] and Gerner et al. [9] (Fig. 1). 


\begin{tabular}{|c|c|c|c|}
\hline \multicolumn{4}{|c|}{ Nomenclature } \\
\hline$A$ & generic flow property & $\beta$ & angle of sideslip \\
\hline$C_{\text {din }}$ & dynamic pressure coefficient & $\beta_{T}$ & angle of sideslip, tangential reference system \\
\hline$C_{\alpha_{T}}$ & angle of attack coefficient (low angles) & $\phi$ & pitch angle, polar reference system \\
\hline$C_{\beta_{T}}$ & angle of attack coefficient (low angles) & $\theta$ & pitch angle, polar reference system \\
\hline$C_{\phi}$ & angle of attack coefficient (high angles) & \multicolumn{2}{|c|}{ Subscripts } \\
\hline$C_{\theta}$ & angle of attack coefficient (high angles) & $i$ & $i$ th data noint in a oiven sector \\
\hline$P$ & probe port pressure & l & nort or sector number $(1-7)$ \\
\hline$V$ & air velocity & $n+$ & adjacent port clockwise to port $n$ \\
\hline$\alpha$ & angle of attack & $n^{-}$ & adjacent port counterclockwise to port $n$ \\
\hline$\alpha_{T}$ & angle of attack, tangential reference system & & \\
\hline
\end{tabular}

In the present paper, an operation technique using a linear interpolation method performed over the data calibration matrices is presented. The performance of this method, in terms of measuring errors, is compared with the traditional one, firstly introduced by Gallington and with later alterations proposed for the calibration/ measuring procedure.

Gallington's method is based upon the fitting of the calibration data (3D matrices where each flow characteristic is a function of two angular dimensionless coefficients) with two dimensional fourth order polynomial functions. Zilliac [10] introduced a new calibration method, using the same dimensionless coefficients as Gallington's, but calculating the flow incidence angles and the static and total pressures, through the local interpolation over the calibration data, applying Akima [11] interpolation method, with a fifth degree polynomial. This technique improved the measurement accuracy, reducing the errors on the determination of the flow angle and velocity magnitude to $1^{\circ}$ and $1 \%$, respectively. Another different approach was presented by Wenger and Devenport [12], who used a two-step method for the probe calibration. The probe coefficients remained the same, but the authors applied an initial least-squares curve fitting to the calibration matrices to represent the smooth trend of the surface, followed by a second step consisting of the construction of error tables containing, for each location, the differences between the experimental calibration points and the correspondent points on the fitting surface.
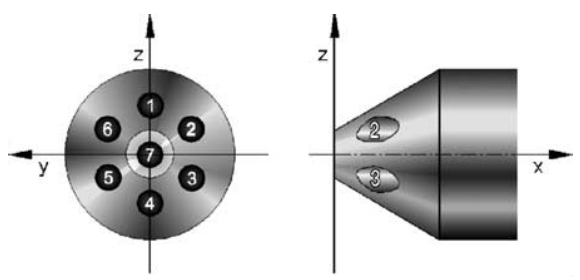

Fig. 1. Seven-hole probe geometry.
An alternative, for the measuring procedure with this type of probe is the use of neural networks software, as presented by Rediniotis et al. [13].

\section{Measurement/calibration procedure}

A flowchart considering different ways of using the seven-hole directional pressure with non-nulling methods for the assessment of the flow characteristics is presented in Fig. 2. The objective of the calibration process is to get the data necessary to be used in a method that allows the calculation of the variables characterizing the flow (two direction angles, total pressure and dynamic pressure) based upon the pressure values measured in the probe's seven taps. For this purpose, the relations between dimensionless coefficients (build up with the measured pressures) and the flow characteristics should be derived. In this work, two of the three different ways, presented in Fig. 2, to determine the flow characteristics were tested and compared (linear interpolation and fourth-order polynomials).

In the calibration process, the probe is submitted to a set of very well characterized incoming flows with the provenience direction defined by the values of two angles. Depending on how large is the angle of incidence of the flow at the probe tip, one of two different systems of coordinates and corresponding sets of coefficients is used.

In the case of flow with low angles of incidence (typically less than $30^{\circ}$ ), for which it does not occur separation at any point of the probe extremity, a tangential referential is used. The angles $\alpha$ and $\beta$ define, in this case, the flow direction (Fig. 3).

The seven pressure values measured at the probe are entered in the calculation of intermediate pressure coefficients that are used to the final determination of two direction-dependent dimensionless coefficients. In addition, two pressure dimensionless coefficients, one related to the total pressure and the other to the dynamic pressure are calculated 
Coefficients for low incidence angles

\begin{tabular}{|c|c|c|c|}
\hline \multicolumn{2}{|c|}{ Intermediate coefficients } & \multirow{2}{*}{$\begin{array}{l}\text { Angular coefficients } \\
C_{x_{T}}=\frac{1}{3}\left(2 C_{\alpha a}+C_{\alpha b}-C_{\alpha c}\right)\end{array}$} & Pressure coefficients \\
\hline$C_{\alpha b}=\frac{P_{3}-P_{6}}{P_{7}-\overline{P_{1-6}}}$ & (1) & & $C_{\mathrm{din}}=\frac{P_{7}-\overline{P_{1-6}}}{P_{\mathrm{tot}}-P_{\mathrm{est}}}$ \\
\hline$C_{\alpha c}=\frac{P_{2}-P_{5}}{P_{7}-\overline{P_{1-6}}}$ & (2) & & \\
\hline$C_{\alpha a}=\frac{P_{4}-P_{1}}{P_{7}-\overline{P_{1-6}}}$ & (3) & $C_{\beta_{T}}=\frac{1}{\sqrt{3}}\left(C_{\alpha b}+C_{\alpha c}\right)$ & $C_{\mathrm{tot}}=\frac{P_{7}-P_{\mathrm{tot}}}{P_{7}-\overline{P_{1-6}}}$ \\
\hline
\end{tabular}

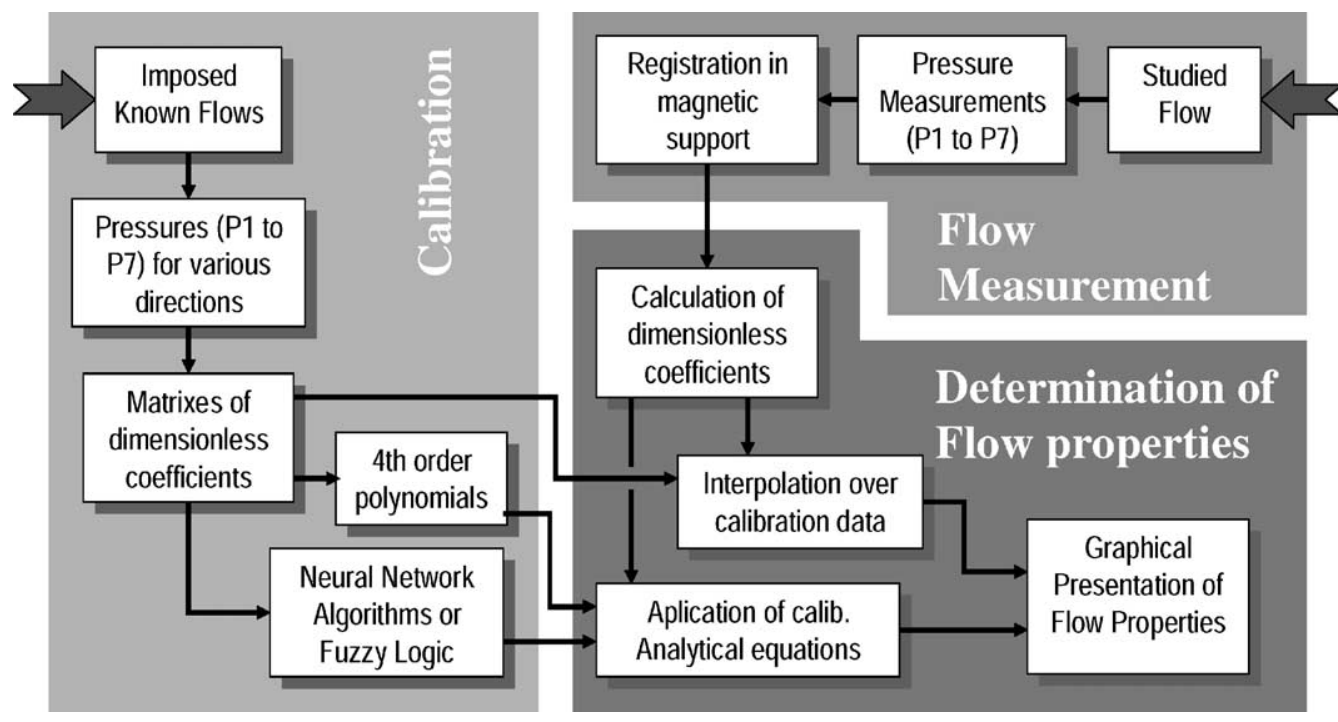

Fig. 2. Flowchart of calibration/measurement processes for non-nulling methods used with directional pressure probes.
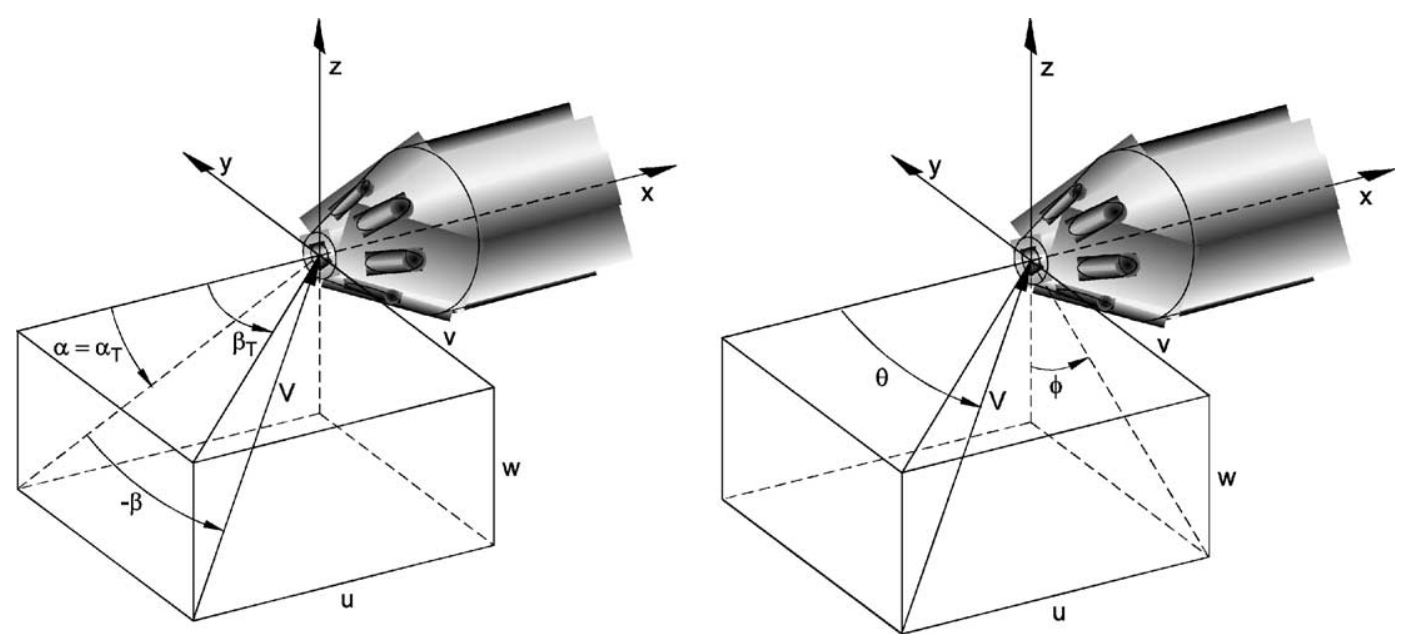

Fig. 3. Systems of axles for low and high incidence angles.

In the former expressions, an overbar is used to indicate the average value of the pressures measured in the holes indicated by the used sub-indices for $P$. For high incidence angles, a polar coordinate referential in which $\phi$ and $\theta$ define the flow's direction is used. In this case, the flow tends to be separated from the probe tip in the 
downstream pressure taps zone. Therefore, the coefficients are only based upon the pressure values in the holes where the flow remains attached, because the values in the other holes are meaningless for identification of the flow direction.

Coefficients for high incidence angles

\begin{tabular}{lll}
\hline Angular coefficients & Pressure coefficients \\
$C_{\phi}=\frac{P_{n^{-}}-P_{n^{+}}}{P_{n}-\left(P_{n^{-}}+P_{n^{+}}\right) / 2}$ & (8) $C_{\mathrm{tot}}=\frac{P_{n}-P_{\mathrm{tot}}}{P_{n}-\left(P_{n^{-}}+P_{n^{+}}\right) / 2} \quad(10)$ \\
$C_{\theta}=\frac{P_{n}-P_{7}}{P_{n}-\left(P_{n^{-}}+P_{n^{+}}\right) / 2}$ & (9) $C_{\mathrm{din}}=\frac{P_{n}-\left(P_{n^{-}}+P_{n^{+}}\right) / 2}{P_{\mathrm{tot}}-P_{\mathrm{est}}}$
\end{tabular}

In those expressions, $n$ refers to the hole with higherpressure value, which points out the general flow direction. The indexes $n^{+}$and $n^{-}$correspond to the two adjacent holes. Thus, seven different groups of nondimensional coefficients are defined, separating the probe measurement field into seven sectors, corresponding to each one of the pressures taps.

The conventional method (fourth-order polynomial fitting equations) introduced by Gallington and Sisson [1] to derive the flow characteristics from the measured pressure data, applies two variables fourth-order polynomials which coefficients $(K)$ are determined from the calibration data. The polynomial coefficients are obtained by solving the sets of equations built up with data obtained from the flows used in the calibration. Thus, to a generic flow property $A$, a polynomial function is built up in the following way:

$$
\begin{aligned}
A= & K_{1}^{A}+K_{2}^{A} C_{\alpha_{T}}+K_{3}^{A} C_{\beta_{T}}+K_{4}^{A} C_{\alpha_{T}}^{2}+K_{5}^{A} C_{\alpha_{T}} C_{\beta_{T}} \\
& +K_{6}^{A} C_{\beta_{T}}^{2}+K_{7}^{A} C_{\alpha_{T}}^{3}+K_{8}^{A} C_{\alpha_{T}}^{2} C_{\beta_{T}}+K_{9}^{A} C_{\alpha_{T}} C_{\beta_{T}}^{2} \\
& +K_{10}^{A} C_{\beta_{T}}^{3}+K_{11}^{A} C_{\alpha_{T}}^{4}+K_{12}^{A} C_{\alpha_{T}}^{3} C_{\beta_{T}}+K_{13}^{A} C_{\alpha_{T}}^{2} C_{\beta_{T}}^{2} \\
& +K_{14}^{A} C_{\alpha_{T}} C_{\beta_{T}}^{3}+K_{15}^{A} C_{\beta_{T}}^{4}
\end{aligned}
$$

As the measuring field of the probe is divided into seven sectors, calibration provides 28 polynomial functions, four for each sector.

The measurement procedure for any flow, the final result of which is the determination of its three velocity components and of dynamic and total pressures, consists on the measurement of the pressure values in the seven pressure taps, followed by the calculation of its suitable dimensionless coefficients and by the selection and resolution of the group of polynomials matching to the corresponding flow sector.

The method introduced in the present work uses a linear interpolation directly performed on the dimensionless coefficients matrices that were generated during the calibration procedure.
As in the conventional method, during calibration, different flow incidences are used according to a direction mesh, previously established, and their suitable non-dimensional coefficients are calculated. These and the matching angular co-ordinates form seven matrices, one for each sector, of the following type.

$$
\left[\begin{array}{cccccc}
\phi_{1} & \theta_{1} & C_{\text {din } 11} & C_{\text {tot } 11} & C_{\phi 11} & C_{\theta 11} \\
\phi_{1} & \theta_{2} & C_{\text {din } 12} & C_{\text {tot } 12} & C_{\phi 12} & C_{\theta 12} \\
\phi_{1} & \theta_{3} & C_{\text {din } 13} & C_{\text {tot } 13} & C_{\phi 13} & C_{\theta 13} \\
\vdots & \vdots & \vdots & \vdots & \vdots & \vdots \\
\phi_{2} & \theta_{1} & C_{\operatorname{din} 21} & C_{\text {tot } 21} & C_{\phi 21} & C_{\theta 21} \\
\phi_{2} & \theta_{2} & C_{\text {din } 22} & C_{\text {tot } 22} & C_{\phi 22} & C_{\theta 22} \\
\vdots & \vdots & \vdots & \vdots & \vdots & \vdots
\end{array}\right]
$$

The determination of the characteristics of an unknown flow starts with the measurement of the seven pressures in the probe and subsequent calculation of related dimensionless coefficients. An example of calculation for a flow with a high incidence angle will be used to facilitate the explanation. In this case, the nondimensional coefficients will be named $C_{\phi}^{*}$ and $C_{\theta}^{*}$. The group of calibration matrices to be used is selected by the index of the pressure tap where the highest-pressure has occurred (Fig. 4).

When graphically plotted (Fig. 5), the calibration matrices matched to the direction coefficients define surfaces in the space. In this spatial representation, the values of the angular coefficients $C_{\phi}^{*}$ and $C_{\theta}^{*}$ determined in a given measure are represented by horizontal planes. The intersections of these planes with the matrices surfaces are two lines $f_{a}$ and $f_{b}$ that, when vertically projected in the base plane $\phi, \theta$, intersect in a point with coordinates $\left(\phi^{*}, \theta^{*}\right)$. The lines $f_{a}$ and $f_{b}$ are numerically determined, point-by-point, through inverse interpolation among the points of the corresponding calibration matrices.

After determination of the point $\left(\phi^{*}, \theta^{*}\right)$, which indicates the incoming flow direction, the dynamic pressure and total pressure dimensionless coefficients, $C_{\mathrm{din}}^{*}$ and $C_{\mathrm{tot}}^{*}$, are obtained by direct interpolation, by entering the coordinates of the point in the respective calibration matrices. Then, the total and dynamic pressure values are computed from Eqs. (6) and (7).

\section{Instrumentation and procedures}

A $200 \mathrm{~mm}$ long probe was used, built with brass tubes, with an external diameter, in its cylindrical part, of $5 \mathrm{~mm}$. For the pressure taps, very thin tubes with a 1 $\mathrm{mm}$ internal diameter were used. They were put inside the external tube, the interstitial spaces among them being fulfilled with solder. The probe conical tip was shaped with a solid angle of amplitude equal to $60^{\circ}$. 

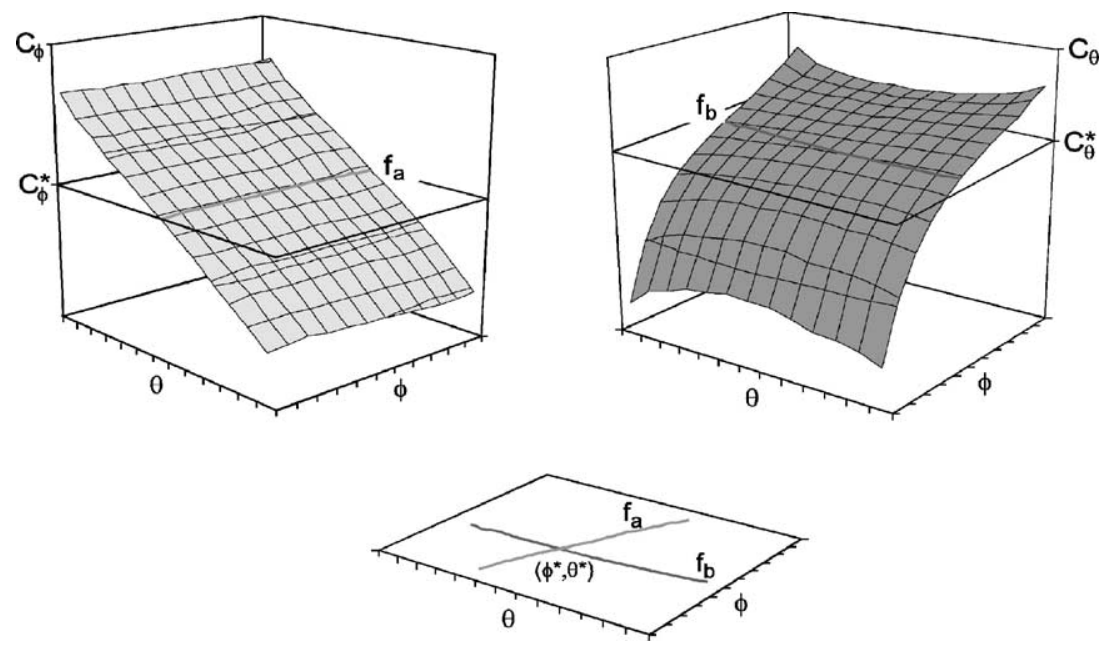

Fig. 4. Determination of flow direction angles.

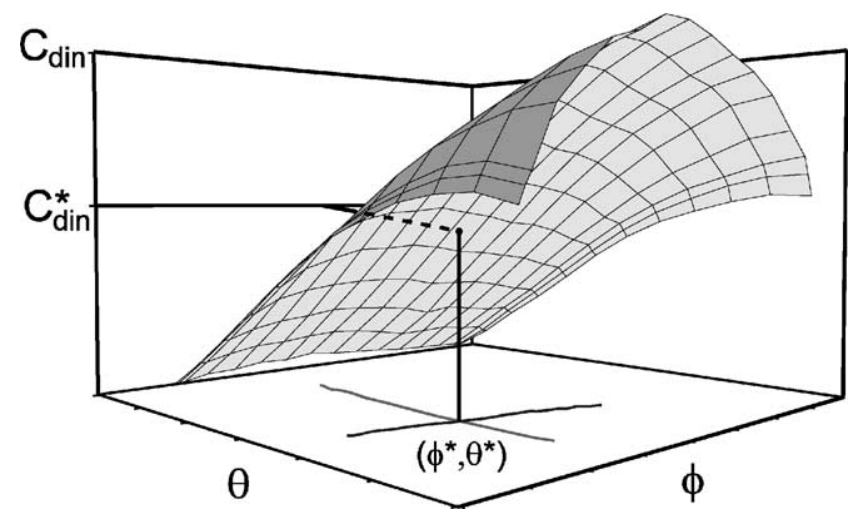

Fig. 5. Determination of dynamic pressure coefficient.

A jet flow, with an initial velocity of $32 \mathrm{~m} / \mathrm{s}$, and a turbulence intensity of $2 \%$, coming from the circular exit section $(d=80 \mathrm{~mm})$ of a circuit comprising a long tube placed after a centrifugal fan, was used as the calibration flow. A manual driven mechanism was made to the probe angular positioning during calibration. The mechanism was designed in such a way that the probe orientation relatively to the flow may be changed while its extremity remains exactly at the same fixed position in the axis of the jet. The acquisition of the pressure values during the calibration was done using a mechanical 48 channels Scanivalve and a computer with an analogue-to-digital conversion interface card (KeithleyMetrabyte DAS 800).

In the calibration procedure, for the central sector of the probe (sector 7), incoming flows were defined by a grid with the direction angles $\alpha_{t}$ and $\beta_{t}$ varying by $5^{\circ}$ steps, from $-20^{\circ}$ to $+20^{\circ}$. In the borders of the domain, the steps were narrowed to $2^{\circ}$. In the case of the peripheral sectors, the angle $\theta$ varied between $20^{\circ}$ and $70^{\circ}$, by steps of $5^{\circ}$, while the angle $\phi$ was varied from $0^{\circ}$ to $360^{\circ}$, generally with increments of $5^{\circ}$. In transition areas between consecutive sectors, the grid was refined using a step angle of $1^{\circ}$ in $\phi$. A computer program written in the TestPoint language automated the acquisition.

\section{Errors assessment}

To allow the assessment of the errors resulting from the use of the direct interpolation method, data about flows with directions corresponding to intermediate points in the two grids of angles were acquired during the calibration procedure. For those flows, which data was not included in the matrices resulting from the calibration, the characteristics were calculated using the aforementioned methods, where the pressures measured in the probe seven ports were the input data. Once the flow characteristics were imposed and, thus, known in advance, it was possible to compute the method errors, subtracting the calculated values from the imposed ones. As, for the same probe, the errors have been previously estimated for the conventional method (Cruz [14]) using a similar procedure, a comparison between the performances of both methods was done.

It was possible to obtain the maps with the distribution of the errors as a function of the incidence angles for all the flow characteristics in all the probe sectors. An example is included in Fig. 6. For flow direction angles, absolute errors are used, while for total and dynamic pressures, relative errors are considered.

A comparison between the conventional method (fourth-order polynomials, 4OP) and the direct interpolation (DI) method can be analysed in Fig. 7. The mean value and the standard deviation of the errors of all the analysed incident flows were calculated for the central sector and for one of the peripheral sectors (number 5). 


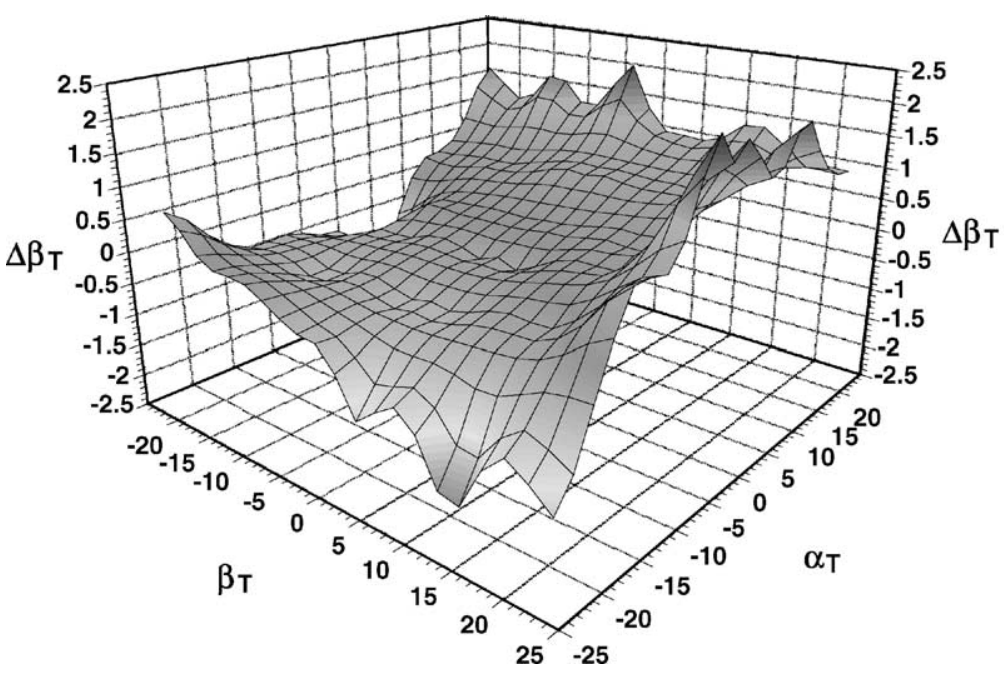

Fig. 6. Distribution of the errors on the angle $\beta_{T}$ in the central sector.

Angle errors on Sector 5 (peripheral)

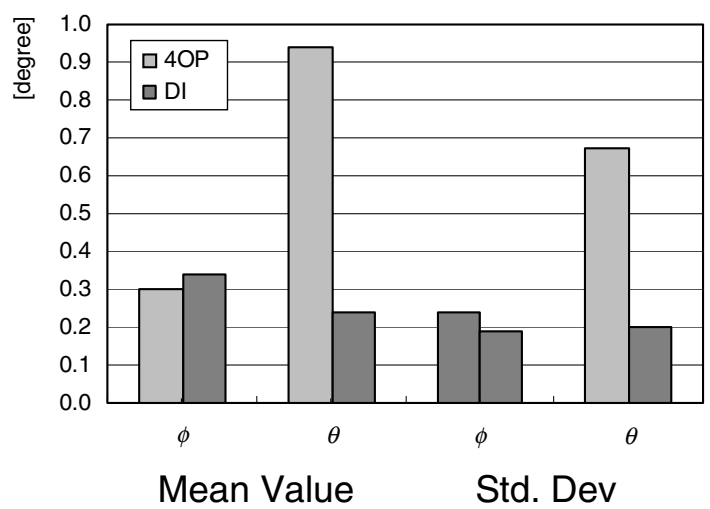

Pressure errors on Sector 5 (peripheral)

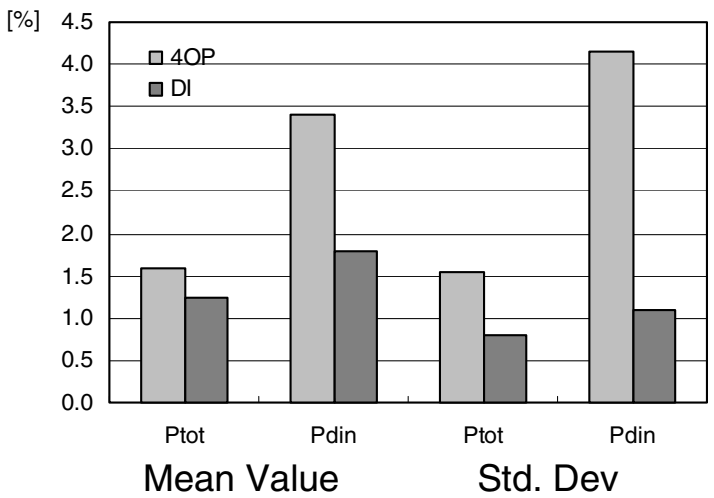

Angle errors on Sector 7 (central)

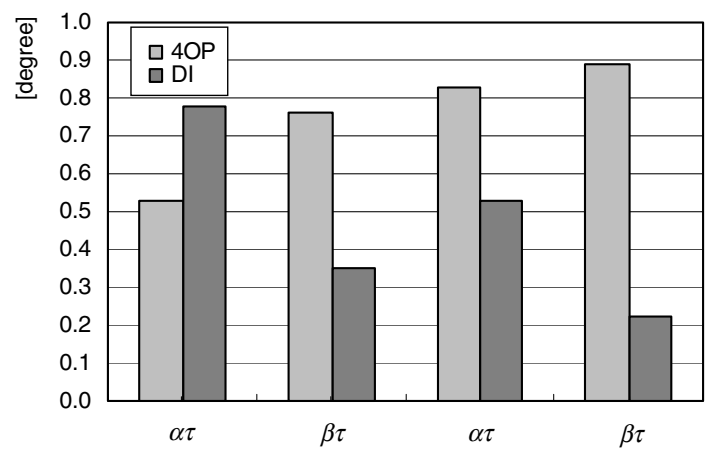

Mean Value

Std. Dev

Pressure errors on Sector 7 (central)

[\%]

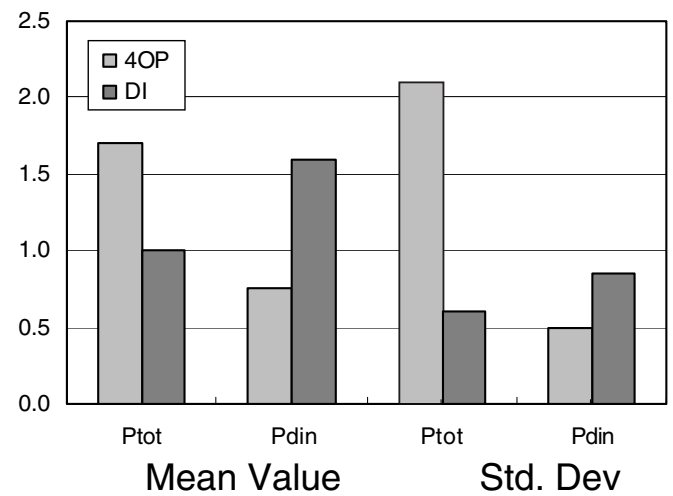

Fig. 7. Mean value and standard deviations of errors on the central sector and on a peripheral sector.

In a general way, the DI method shows higher precision and results uniformity than the 4OP method. In the central sector, the errors in angle $\beta_{t}$ show a clear decrease in the DI method relatively to the $4 \mathrm{OP}$ method, from $0.76^{\circ}$ to $0.35^{\circ}$, in the mean value. In addition, the dispersion of the errors is reduced, e.g. the standard deviation falls from $0.89^{\circ}$ to $0.22^{\circ}$ and the maximum error falls from $3.51^{\circ}$ to $0.71^{\circ}$ (data not presented 
graphically). As regards the angle $\alpha_{t}$, the mean value of the error is larger for the DI method $\left(0.79^{\circ}\right)$ than for the 4OP method $\left(0.52^{\circ}\right)$, but, by the other hand, the standard deviation has an opposite behaviour ( 0.56 for DI and 0.82 for $4 \mathrm{OP}$ ).

In the peripheral sector, for the angle $\theta$, there is a substantial decrease for the DI method, either in the mean value of the error $\left(0.94-0.24^{\circ}\right)$, or in the standard deviation $\left(0.67-0.20^{\circ}\right)$. The improvement on the behaviour of the probe, when using the DI method, is reinforced by the data concerning the maximum value of the error on angle $\theta$, i.e. a reduction from $3.25^{\circ}$ to $0.85^{\circ}$. In terms of the errors on angle $\phi$, there is a slight increase in the mean value and a decrease in the standard deviation, when comparing DI and 4OP methods, respectively. In the maximum value of the error, DI method still performs better $\left(1.22^{\circ}\right.$ for $4 \mathrm{OP}$ and $0.76^{\circ}$ for DI).

Relatively to the errors in the dynamic pressure and total pressure measurements, DI method presents, in an overall view, better results than the 4OP method. There is only one exception, on the dynamic pressure in the central sector, but, in this case, the differences between the two methods are not very significant, because both present a close behaviour.

\section{Wind tunnel experiments}

Wind tunnel experiments were carried out to test the developed tools for the presented calibration/measurement method. Those, here presented, were obtained with a flow field mapping system, developed by the authors
[15] to be installed in a wind tunnel belonging to the Portuguese Air Force Academy. The system is based on a seven-hole directional pressure probe and has the possibility of scanning all the wind tunnel test chamber, with a $1.3 \mathrm{~m} \times 0.8 \mathrm{~m}$ cross-section and a length of $2 \mathrm{~m}$, working in a flow velocity ranging from 3 to $70 \mathrm{~m} / \mathrm{s}$ (Fig. 8).

Five INA MLFI translation units, using a guided moving car connected to a toothed belt, were used in the construction of the traversing mechanism. All of them stay over and outside of the wind tunnel test chamber, where the air velocity can reach $70 \mathrm{~m} / \mathrm{s}$, the probe and its support (an aluminium symmetrical wing profile) being the only parts penetrating in the flow. The spatial resolution of the positioning mechanism is $0.435 \mathrm{~mm}$, on the $X$ - and $Z$-axes, and $0.084 \mathrm{~mm}$, on the $Y$-axis.

The step motors are controlled by a unit composed by two indexer cards (RS 440-098) and three drivers (RS 217-3627), which communicates with the computer through the RS232 serial port. A Keithley-Metrabyte DAS 800 plug-in board was used as interface, assuring the analogue to digital conversion of the probe pressure signals and the digital output used to control a Scanivalve pressure scanning system, to which the seven pressure lines of the probe are connected. The pressure output signal of the Scanivalve is measured by a differential piezo-resistive pressure sensor, Omega PX $16 \mathrm{O}$.

A flow field survey of the space over a wing profile, in terms of the velocity and static pressure, for a free stream velocity equal to $40 \mathrm{~m} / \mathrm{s}$, is depicted in the Fig. 9, where FlowVis, a software tool developed by Lopes [16] for the post-processing of scalar and vectorial fields, was used.

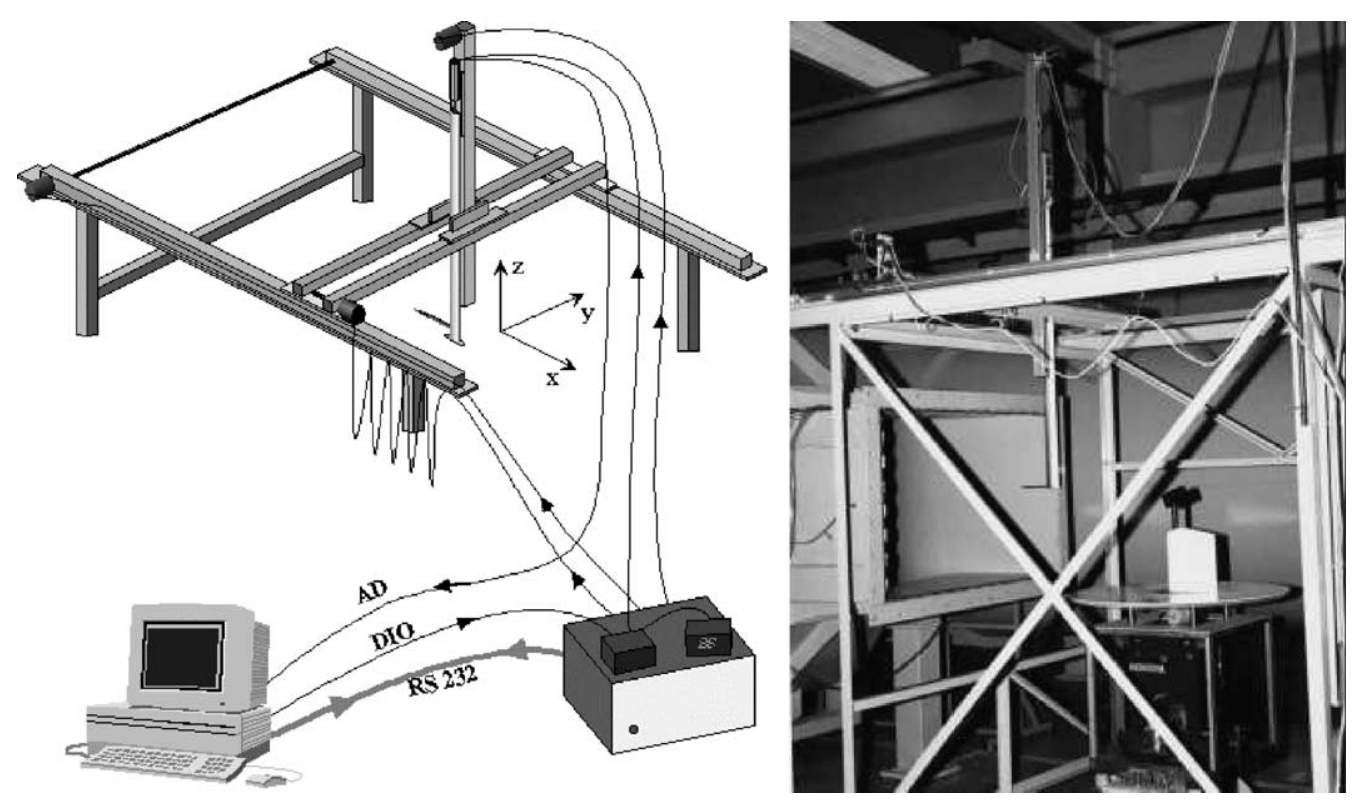

Fig. 8. Traversing mechanism scheme and photography of the probe in the wind tunnel test chamber. 


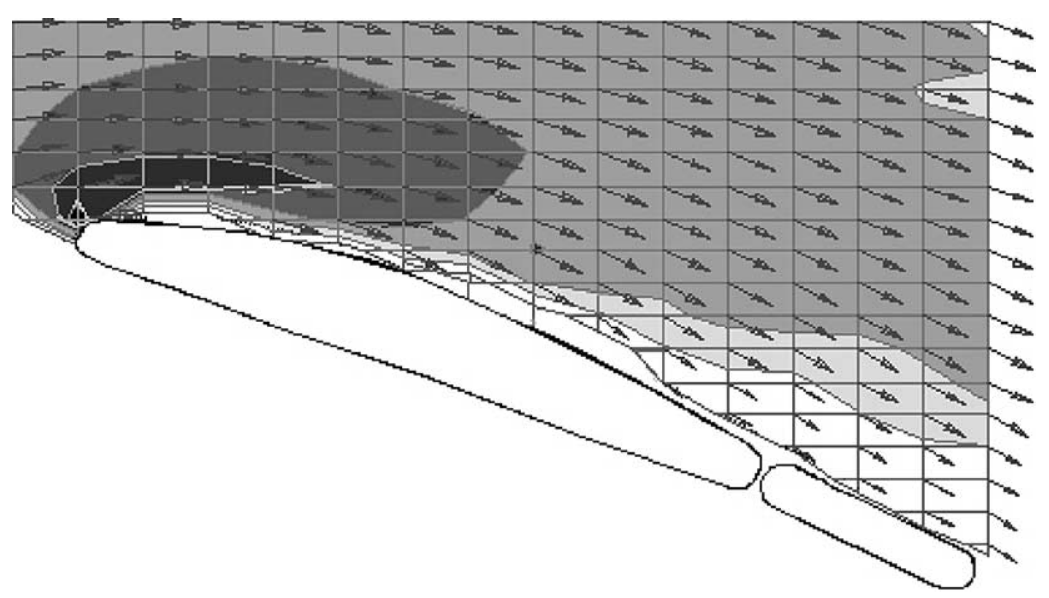

Fig. 9. Mapping of velocity and pressure fields over a wing profile.

\section{Conclusion}

The use of the linear interpolation method on the seven-hole probe calibration proved to be an option that improves the measurement precision compared to the initial Gallington method based on the two dimensional fourth-order polynomials fitting of calibration data. The inaccuracy errors found for angular and magnitude quantities, less than $0.8^{\circ}$ and $1.5 \%$ respectively, are of the same order as those found by Zilliac [10], with a method based being upon local interpolation through the Akima method, and Wenger and Devenport [12], who use a two-step interpolation method.

It is expected that results can still be enhanced if a higher number of calibration points is used, mainly on the transition areas between sectors. In addition, the use of a higher precision rotating mechanism in the angular positioning of the probe during the calibration procedure can improve the quality of the results.

The inter-connection of the different software tools used for data-acquisition, data reduction and postprocessing was done successfully, having as a result meaningful pictures with the mapping of the different flow-field physical variables.

Nevertheless, the incapability of the probe to measure flows with very low velocity or very high incidence angles $\left(>70^{\circ}\right)$ is clear and should be stressed.

\section{References}

[1] R. Gallington, G. Sisson, Flow visualization using a computerized data acquisition system, in: International Symposium on Flow Visualization, Bochum, 9-12 September 1980.

[2] J.E. Hackett, A. Sugavanam, Evaluation of a complete wake integral for the drag of a car-like shape, SAE Paper 840577, 1984.
[3] J.E. Hackett, J.E. Williams, J. Patrick Jr., Wake traverses behind production cars and their interpretation, SAE Paper 850280, 1985.

[4] A. Cogotti, Prospects for aerodynamic research in the Pininfarina wind tunnel, SAE Paper 905149, 1990.

[5] A. Cogotti, Car wake imaging using a seven-hole probe, SAE Paper 860214, 1986; Aerodynamics: recent developments, SAE SP-656.

[6] M.C.G. Silva, D.X. Viegas, Calibration and use of a non-nulling seven-hole pressure probe, AGARD Conference Proceedings 535 (1994) 3.1-3.7.

[7] C. Ostowari, W.H. Wentz Jr., Modified calibration technique of a five-hole probe for high angle flows, Experiments in Fluids 1 (1983) 166-168.

[8] K.N. Everett, A.A. Gerner, D.A. Durston, Seven-hole cone probes for high angle flow measurement: theory and calibration, AIAA Journal 21 (7) (1983) 992-998.

[9] A.A. Gerner, C.L. Maurer, R. Gallington, Non-nulling seven-hole probes for high angle flow measurement, Experiments in Fluids 2 (1984) 95-103.

[10] G.G. Zilliac, Modelling, calibration, and error analysis of sevenhole pressure probes, Experiments in Fluids 14 (1993) 104 120 .

[11] H. Akima, A method of bivariate interpolation and smooth surface fitting for irregularly distributed points, ACM Transactions on Mathematical Software 2 (4) (1978) 148.

[12] C.W. Wenger, W.J. Devenport, Seven-hole pressure probe calibration method utilizing look-up error tables, AIAA Journal 37 (6) (1999)

[13] O.K. Redionotis, G. Chryssanthakopoulos, Application of neural networks and fuzzy logic to the calibration of the seven-hole probe, Journal of Fluids Engineering 120 (1998) 95-101.

[14] J.M.S. Cruz, Análise de escoamentos com sonda de sete furos, M.Sc. Thesis, Faculdade de Ciências e Tecnologia da Universidade de Coimbra, 1998 (in Portuguese).

[15] M.C.G. Silva, A.M.G. Lopes, C.A.C. Pereira, J.M.S. Cruz, M.L.S. Mateus, A wind tunnel flow field mapping system based on a seven-hole pressure probe, in: Conference Controlo 98, Coimbra, 9-11 September 1998.

[16] A.M.G. Lopes, FlowViz - a CAD based solution for the graphical post-processing of scalar and vectorial data, Environmental and Modelling Software 12 (1997) 161-168. 